Bundesgesundheitsbl 2021 · 64:304-313 https://doi.org/10.1007/s00103-021-03277-1 Eingegangen: 28. April 2020

Angenommen: 17. Dezember 2020

Online publiziert: 26. Januar 2021

(c) Springer-Verlag GmbH Deutschland, ein Teil von Springer Nature 2021

\author{
Ernst G. Pfenninger ${ }^{1} \cdot$ Pascal Christ $^{2} \cdot$ Martin Neumüller $^{2} \cdot$ Alexander Dinse- $^{2}$ \\ Lambracht $^{3,4}$ \\ 'Stabsstelle Katastrophenschutz, Universitätsklinikum Ulm, Ulm, Deutschland \\ ${ }^{2}$ Stabsstelle Sicherheit, Universitätsklinikum Ulm, Ulm, Deutschland \\ ${ }^{3}$ Zentrale Interdisziplinare Notaufnahme, Universitätsklinikum Ulm, Ulm, Deutschland \\ ${ }^{4}$ Interdisziplinäres Notfallzentrum, Kliniken Aurich-Emden-Norden, Emden, Deutschland
}

\title{
Beurteilung des Infektionsrisikos durch SARS-CoV-2 für medizinisches Personal - Erkenntnisse aus der Praxis
}

\section{Hintergrund}

Das Virus SARS-CoV-2 hat sich nach seiner erstmaligen Entdeckung im Dezember 2019 in kurzer Zeit als Erreger der Erkrankung COVID-19 sehr effizient durch Tröpfchen- und Aerosolinfektion von Mensch zu Mensch in der Bevölkerung ausgebreitet. Bis Ende August 2020 sind weltweit über 24 Mio. Infizierte und fast 850.000 Tote bei der Weltgesundheitsorganisation (WHO) gemeldet [1]. In der internationalen Laienpresse wird das Risiko für medizinisches Personal, an COVID-19 zu erkranken, als hoch eingeschätzt [2]. In China entfielen $1716(3,8 \%)$ von 44.672 mit PCRbestätigten COVID-19-Erkrankten, die bis zum 11.02.2020 diagnostiziert wurden, auf Beschäftigte im Gesundheitswesen [3]. In den USA fand eine Studie, dass $11 \%$ aller COVID-19-Erkrankten zum medizinischen Personal gehören [4]. Eine australische Simulationsstudie zeigte, dass ein infizierter Patient in der Notaufnahme einer Klinik 6 bis 14 Personalkontakte pro Tag hat [5]. Laut Robert Koch-Institut (RKI) fanden bis Ende August 2020 in deutschen Krankenhäusern 402 COVID-19-Ausbrüche statt, wobei im Schnitt 10,2 Patient*innen angesteckt wurden [6]. Eine Ansteckung kann direkt über die Schleimhäute der Atemwege er-

Bei Veröffentlichung des Artikels stehen mindestens zwei Impfstoffe zur Verfügung. folgen oder auch indirekt über Hände, die mit Mund- oder Nasenschleimhaut sowie der Augenbindehaut in Kontakt gebracht werden [7]. Für die Übertragung kommen nicht nur akut COVID-19-Erkrankte, sondern auch symptomlose Personen in Betracht. Das Infektionsrisiko steigt mit der Anzahl und der Dauer der ungeschützten Kontakte. Die Inkubationszeit liegt im Mittel (Median) bei 5 bis 6 Tagen (Spannweite 1 bis 14 Tage; [8]).

Anfangs war es noch umstritten, ob eine Übertragung auch durch Aerosole stattfinden kann [9]. Nach Aussage der National Academies of Sciences, Engineering, and Medicine können Aerosole infektiöses Material enthalten [10]. Aerosole entstehen bei Husten, Niesen und auch beim Sprechen oder Singen. Eine Übertragung über unbelebte Oberflächen ist bisher nicht dokumentiert. Eine Infektion mit SARS-CoV-2 über Oberflächen, die nicht zur direkten Umgebung eines symptomatischen Patienten gehören, wie z. B. importierte Waren, Postsendungen oder Gepäck, erscheint daher unwahrscheinlich. Eine Übertragung durch kontaminierte Oberflächen ist jedoch in der unmittelbaren Umgebung der infektiösen Person nicht auszuschließen, da vermehrungsfähige SARS-CoV-2-Viren unter Laborbedingungen auf Flächen einige Zeit infektiös bleiben können [7].

Im Folgenden soll angesichts wieder steigender Infektionszahlen [11] das nach dem gegenwärtigen Stand der
Kenntnis (August 2020) bekannte Risiko, an COVID-19 zu erkranken oder $\mathrm{zu}$ versterben, für das Personal in der Notaufnahme und weiteren Bereichen einer Klinik betrachtet werden.

\section{Biologische Gefährdung}

Durch Beschluss des Ausschusses für Biologische Arbeitsstoffe (ABAS) wurde das Virus SARS-CoV-2 der Risikostufe 3 gemäß Technischer Regeln für Biologische Arbeitsstoffe (TRBA 462) zugeordnet [8]. Nach $\$ 3$ Biostoffverordnung sind Biostoffe der Risikogruppe 3 als folgend definiert: „Biostoffe, die eine schwere Krankheit beim Menschen hervorrufen und eine ernste Gefahr für Beschäftigte darstellen können; die Gefahr einer Verbreitung in der Bevölkerung kann bestehen, doch ist normalerweise eine wirksame Vorbeugung oder Behandlung möglich" [12].

Die durch SARS-CoV-2 hervorgerufene Erkrankung, die sich vor allem im Rachenraum und der Lunge zeigt, wird von der WHO unter dem Namen COVID-19 geführt. Aktuell steht keine Schutzimpfung gegen SARS-CoV-2 zur Verfügung. Studien haben gezeigt, dass Personen nach durchgemachter SARSCoV-2-Infektion spezifische Antikörper entwickeln. Unklar ist, wie regelhaft, robust und dauerhaft dieser Immunstatus aufgebaut wird.

Biologische Gefährdungen im klinischen Bereich liegen überall dort vor, wo 


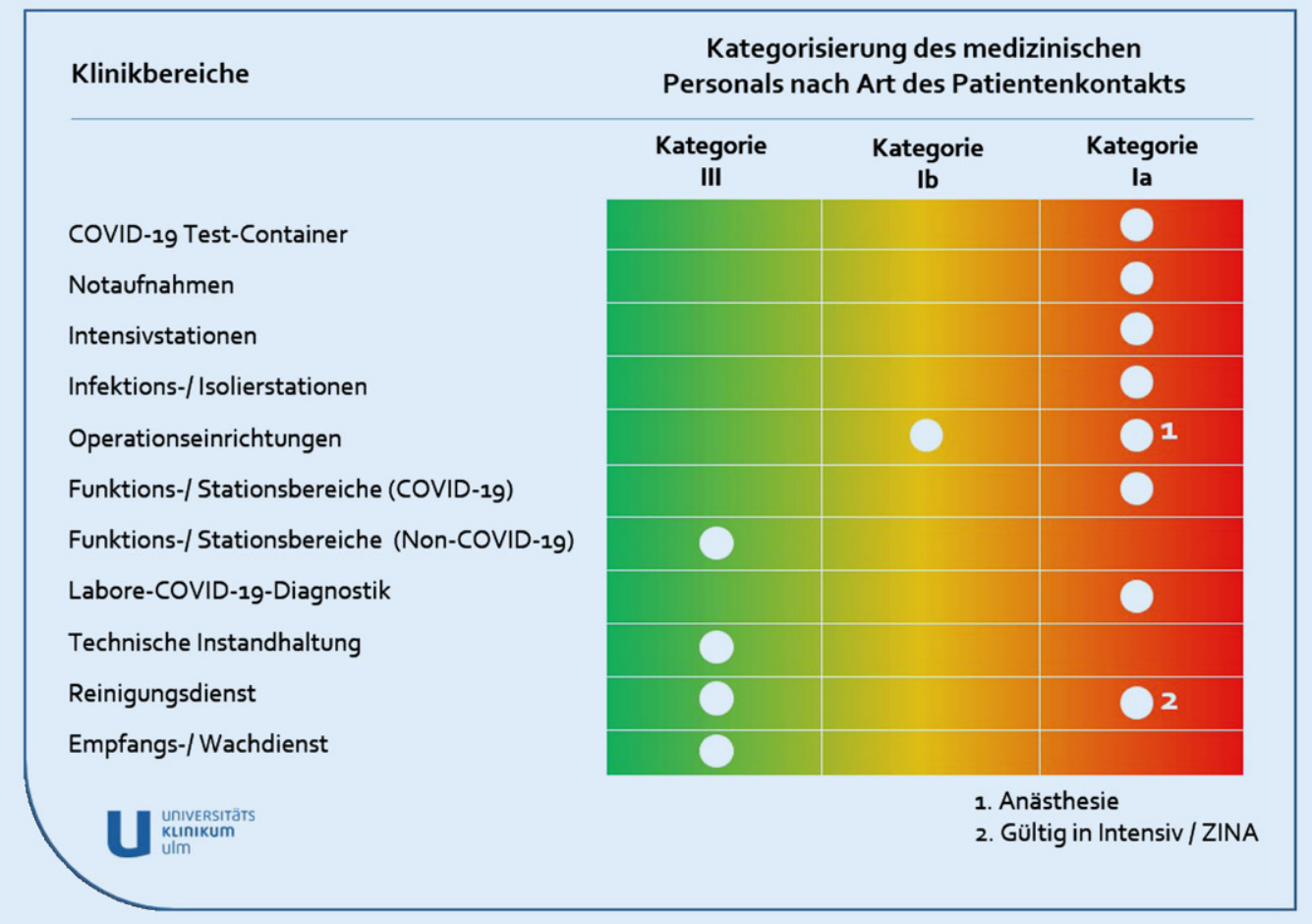

Abb. $1 \varangle$ Infektionswahrscheinlichkeit ohne persönliche Schutzausrüstung in verschiedenen Klinikbereichen. Die Infektionswahrscheinlichkeit wird aus der Kategorisierung des Robert Koch-Instituts (RKI) zu Patientenkontakten des medizinischen Personals [13] sowie der Einschätzung der Autoren abgeleitet. Kategorien: $l a$ - hohes Expositionsrisiko, $l b$ - begrenztes Expositionsrisiko, III-geringes Expositionsrisiko. Einstufung: rot - hohes Expositionsrisiko, gelb - mittleres Expositionsrisiko, grün - geringes Expositionsrisiko

ambulant oder stationär behandelte $\mathrm{Pa}$ tient*innen potenziell oder bestätigt infektiös sind. Darüber hinaus sind Mitarbeiter ${ }^{\star}$ innen gefährdet, die mit potenziell infektiösem humanen Probenmaterial umgehen oder im Rahmen von Instandhaltungs- und Reinigungsarbeiten in Kontakt gelangen können.

\section{Infektionswahrscheinlichkeit ohne spezielle persönliche Schutzausausrüstung (PSA)}

Gemäß RKI wird medizinisches Personal anhand seiner Patientenkontakte in folgende Kategorien eingeteilt [13]:

Kategorie Ia. Medizinisches Personal mit hohem Expositionsrisiko, z. B. ungeschützte relevante Exposition zu Sekreten oder Exposition gegenüber Aerosolen von COVID-19-Fällen (z. B. im Rahmen von Intubation oder Bronchoskopien).

Kategorie Ib. Medizinisches Personal mit begrenztem Expositionsrisiko, z. B. mit Kontakt unter 2 Metern zu COVID19-Fällen ohne Schutzausrüstung, länger als $15 \mathrm{~min}$ Face-to-Face-Kontakt; grundsätzlich gilt: Je länger und enger der Kontakt, desto höher das Risiko.

Kategorie III. Medizinisches Personal ohne Schutzausrüstung und mit Distanz über 2 Metern sowie bei nicht zu erwartender Exposition gegenüber Sekreten, Körperflüssigkeiten oder Aerosolen; bei Verwendung von Schutzausrüstung auch unter $2 \mathrm{~m}$ Abstand.

Aus dieser Kategorisierung ergibt sich, dass in einer Klinik in den verschiedensten Bereichen die Möglichkeit einer Infektion mit SARS-CoV-2 in unterschiedlichem Maß gegeben ist (• Abb. 1):

\section{Kategorie la}

Ein hohes Expositionsrisiko aufgrund eines Kontaktes mit Sekreten, Aerosolen und Körperflüssigkeiten von COVID19-Fällen besteht für den COVID-19Testcontainer, Notaufnahmen, Intensiv-/ Isolierstationen, Funktions-/Stationsbereiche mit COVID-19-Patienten sowie für die Anästhesie in Operationseinrichtungen. Für den Reinigungsdienst ist die Beurteilung des Risikos davon abhängig, wo Reinigungs-/Dienstleistungen durchgeführt werden. In Bereichen der
Notaufnahmen, Intensiv- und Infektionsstationen sowie Normalstationen mit COVID-19-Patienten kann ein Infektionsrisiko der Kategorie Ia bestehen. Diese Einstufung gilt auch für die Labore der COVID-19-Diagnostik.

\section{Kategorie Ib}

Sofern Operationssäle mit turbulenzarmer Verdrängungsströmung (TAV-Decken) ausgestattet sind, ist von einer Minderung des Expositionsrisikos auszugehen [14]. Mittels technischer Maßnahmen erscheint eine Einstufung nach Kategorie Ib sinnvoll.

\section{Kategorie III ${ }^{1}$}

Gemäß RKI wird medizinisches Personal, welches keine spezielle Schutzausrüstung verwendet und in Bereichen der Patientenversorgung die bestätigten NonCOVID-19-Patienten betreut, der Kategorie III zugeordnet. Eingeschlossen sind in der Kategorie III auch Personengrup-

\footnotetext{
1 Kategorie II ist in der zitierten Quelle nicht aufgeführt. Die zitierte Quelle wurde zwischenzeitlich geändert.
} 
pen, die keiner Exposition von Sekreten, Körperflüssigkeiten oder Aerosolen ausgesetzt sind oder generell eine Distanz $>2 \mathrm{~m}$ einhalten.

\section{Beurteilung des Infekti- onsrisikos nach offizieller Statistik}

Aus den täglich vom RKI veröffentlichten Zahlen zu Infektionen und Todesfällen zu COVID-19 lässt sich anhand des Verlaufs über 4 Monate eine tatsächliche Einschätzung der Gefährdung des medizinischen Personals vornehmen (• Tab. 1).

Die Infektionsrate des medizinischen Personals mit SARS-CoV-2 stieg im Vergleich zur Gesamtbevölkerung von ursprünglich $73 \%$ im April auf $94 \%$ im August 2020 an (• Tab. 1) und würde damit nun ungefähr der in der Gesamtbevölkerung entsprechen, ungeachtet dessen, dass medizinisches Personal durch bessere medizinische Vorbildung und entsprechende Schutzmaßnahmen eigentlich besser gegen Infektionen geschützt sein sollte. Allerdings ist dem gegenüberzustellen, dass medizinisches Personal im Gegensatz zur Allgemeinbevölkerung einem Vielfachen an Kontakten und auch Kontaktzeiten mit an COVID-19 Erkrankten ausgesetzt ist und damit die Gefährdung und Infektionswahrscheinlichkeit sehr hoch ist $[5,18]$. Auch wird medizinisches Personal deutlich häufiger auf eine SARS-CoV-2-Infektion getestet, sodass, im Gegensatz zur Allgemeinbevölkerung, mehr asymptomatisch Infizierte erkannt werden. Zudem ist nicht bekannt, ob sich das medizinische Personal in Zusammenhang mit seiner beruflichen Tätigkeit oder außerhalb derer infiziert hatte $[11,16,17]$. Diesen Aspekt unterstreichen auch Jeremias und Mitarbeiter, die fanden, dass in einer Klinik der Grundversorgung kein Unterschied an Infizierten zwischen Bereichen mit hohem und niedrigem Infektionspotenzial zu finden war [19]. Allerdings fanden die Autoren auch, dass die Rate an infiziertem Personal in der Klinik deutlich geringer als außerhalb des Krankenhauses war (9,9\% gegen 16,7\%). Im Gegensatz zu anderen Ländern ist die vom RKI angegebene Infektionsrate des medizinischen Personals in Deutschland deutlich

Bundesgesundheitsbl 2021 · 64:304-313 https://doi.org/10.1007/s00103-021-03277-1

(c) Springer-Verlag GmbH Deutschland, ein Teil von Springer Nature 2021

\section{E. G. Pfenninger $\cdot$ P. Christ $\cdot$ M. Neumüller $\cdot$ A. Dinse-Lambracht}

\section{Beurteilung des Infektionsrisikos durch SARS-CoV-2 für medizinisches Personal - Erkenntnisse aus der Praxis}

\section{Zusammenfassung}

Das SARS-CoV-2-Virus als Erreger der COVID19-Erkrankung hat sich innerhalb kurzer Zeit weltweit in der Bevölkerung ausgebreitet. Bei der Abfassung des Beitrags stehen noch keine Schutzimpfung und keine spezifische Therapie gegen SARS-CoV-2 zur Verfügung. Bei Veröffentlichung des Artikels werden mindestens zwei Impfstoffe zur Verfügung stehen. In der internationalen Laienpresse wird das Risiko für medizinisches Personal, an SARS-CoV-2 zu erkranken, als hoch eingeschätzt; das Robert Koch-Institut stuft das Risiko für die Gesamtbevölkerung in Deutschland als "hoch" ein. Das Ziel des vorliegenden Beitrags ist es, das Infektionsund Erkrankungsrisiko für medizinisches Personal basierend auf Praxiserfahrungen, nationalen Verordnungen und Richtlinien sowie Infektionszahlen zu diskutieren und neu einzuschätzen. Dabei wird sowohl ungeschütztes als auch mit persönlicher Schutzausrüstung (PSA) ausgestattetes medizinisches Personal betrachtet. Eine entsprechende Risikomatrix wird erstellt. Das Infektionsrisiko für ungeschütztes medizinisches Personal entspricht dem in der Gesamtbevölkerung und ist mit "hoch" einzustufen. Mit entsprechender persönlicher Schutzausrüstung ist das Infektionsrisiko für medizinisches Personal dagegen als "mittel" einzuschätzen. Zur PSA gehören ein flüssigkeitsdichter Schutzkittel, Handschuhe, Maske - Schutzstufe FFP2 oder FFP3 - bei Tätigkeiten mit Tröpfchen oder Aerosolbildung, Kopfhaube und eine geeignete Schutzbrille. Ungenügende Handhygienemaßnahmen, falsche Handhabung der Schutzkleidung sowie lange Dienstzeiten erhöhen das Infektionsrisiko.

Schlüsselwörter

Pandemie · COVID-19 · Infektionsrate Medizinisches Personal · Risikomatrix

\section{Assessment of the risk of infection from SARS-CoV-2 for healthcare workers-findings from practice}

\section{Abstract}

SARS-CoV-2 has rapidly spread over the world in a pandemic manner causing an infection of predominantly pulmonary manifestation named the COVID-19 disease. Currently, there is neither an effective vaccination nor a specific therapy available. At least two vaccines will be available at the time of publication. In the international press, the risk for medical personnel of SARS-CoV-2 is rated as high. The Robert Koch Institute, Germany's leading epidemiological authority, regards the risk of infection for the general population to be high. The aim of this article is to discuss and reassess the risk of infection and disease for healthcare workers based on practical experience, national regulations and guidelines, and the number of infections. Both unprotected healthcare workers and healthcare workers equipped with personal protective equipment (PPE) are considered. A corresponding risk matrix is created. The risk of infection with SAR-CoV-2 for healthcare workers is comparable to the general population and rated as high. Proper use of PPE reduces this risk to medium. PPE consists of liquid-proof gowns, gloves, and filtering face pieces (FFP; FFP 2 as a standard, FFP 3 for aerosol-releasing interventions), a hair cover, and protective goggles. Improper use of PPE, inadequate hygienic measures, and long working shifts increase the risk of infection.

\section{Keywords}

Pandemic · COVID-19 - Infection rate $\cdot$ Medical staff · Risk matrix niedriger: „In Brescia province, the center of Italy's outbreak, 10 to 15 percent of doctors and nurses have been infected and put out of commission, according to a doctor there“, und weiter: „Out of Spain's 40.000 confirmed coronavirus cases, 5.400 - nearly 14 percent - are medical professionals“ $[2,19]$.

Die Todesrate des medizinischen Personals in Deutschland beträgt nur ca. $1 / 10$ bis ein 1/30 gegenüber der Allgemeinbevölkerung. Dies ist sicherlich 
Tab. 1 Angaben zu Infektionen und Todesfällen beim medizinischen Personal (MP) und in der Gesamtbevölkerung (GBV) in Deutschland (an/mit COVID-19 erkrankt) über 4 Monate. Die Angaben bezogen auf 100.000 Einwohner berechnen sich unter Annahme einer Bevölkerung Deutschlands von 83 Mio. und von 5,7 Mio. Beschäftigten im Gesundheitswesen. (Quellen: [11, 15-17])

\begin{tabular}{|c|c|c|c|}
\hline Stichtag & 16.04 .2020 & 18.06.2020 & 21.08.2020 \\
\hline $\begin{array}{l}\text { Infiziertes medizinisches Personal } \\
\text { (MP) }\end{array}$ & $6359(112 / 100.000)$ & $\begin{array}{l}13.602 \\
(239 / 100.000)\end{array}$ & $\begin{array}{l}14.825 \\
(260 / 100.000)\end{array}$ \\
\hline Davon verstorben & $8(0,13 \%)$ & $20(0,15 \%)$ & $23(0,16 \%)$ \\
\hline $\begin{array}{l}\text { Infizierte Gesamtbevölkerung } \\
\text { (GBV) }\end{array}$ & $\begin{array}{l}130.450 \\
(157 / 100.000)\end{array}$ & $\begin{array}{l}187.764 \\
(226 / 100.000)\end{array}$ & $\begin{array}{l}230.048 \\
(277 / 100.000)\end{array}$ \\
\hline Davon verstorben & $3569(2,74 \%)$ & $8856(4,72 \%)$ & $9260(4,03 \%)$ \\
\hline Infektionen MP/GBV pro 100.000 & $73 \%$ & $94 \%$ & $94 \%$ \\
\hline Verstorben MP/GBV pro 100.000 & $5 \%$ & $3 \%$ & $4 \%$ \\
\hline
\end{tabular}

unter anderem dem deutlich niedrigeren Durchschnittsalter mit einem Median von 41 Jahren der im medizinischen Bereich Tätigen [11] und den damit verbundenen milderen Verlaufsformen der Erkrankung geschuldet [17]. Spekulativ könnte auch ein besseres Wissen um Symptome und damit eventuell eine frühzeitigere medizinische Behandlung eine Rolle spielen. Des Weiteren könnte spekuliert werden, dass durch die korrekte Verwendung einer PSA die Virusbelastung des Personals und damit die „Infektschwere“ geringer sei, zu beiden Annahmen liegen jedoch keine wissenschaftlichen Daten vor.

\section{Beurteilung des Infektionsrisi- kos bei korrekter Verwendung geeigneter persönlicher Schutzausrüstung (PSA)}

Eine kürzlich erschienene Studie aus Massachusetts/USA zeigte, dass am Anfang der Pandemie von 9850 getesteten Mitarbeitenden ohne Mund-NasenSchutz (MNS) 12,9\% positiv waren, mit einem gewichteten mittleren Anstieg von $1,16 \%$ pro Tag bis auf über $21 \%$. Nachdem für Personal und Patienten das Tragen eines chirurgischen MNS bei Betreten der Klinik eingeführt wurde, sank die Infektionsrate des medizinischen Personals linear von $14,46 \%$ auf $11,46 \%$ mit einem gewichteten Mittelwert linear um $0,49 \%$ pro Tag [20]. In einem Editorial hierzu in der Zeitschrift JAMA wurde betont, dass durch das Tragen eines MNS vor allem die Ausbreitung des Virus durch asymptomatische Träger vermindert werden würde: „a cloth face covering can help reduce the spread of COVID-19 from asymptomatic individuals or others" [21].

Über einen ähnlichen Schutzeffekt berichtete eine indische Arbeitsgruppe für Gesichtsschutzschilde. Vor Benutzung von Gesichtsschutzschilden infizierten sich $19 \%$ des medizinischen Personals bei Kontakt mit 222 infizierten Patienten, nach Einführung von Gesichtsschutzschilden niemand mehr bei Kontakt mit 2682 positiv getesteten Patienten [21]. Allerdings betonen die für Deutschland zuständigen Arbeitsausschüsse beim Bundesministerium für Arbeit und Soziales (BMAS): „Aufgabe eines Gesichtsschutzschildes ist, den Träger/die Trägerin gegen Gefahren von außen zu schützen (Eigenschutz). Eine Filterwirkung ist nicht gegeben" [23]. RKI und die Arbeitsausschüsse beim BMAS empfehlen für medizinisches Personal beim direkten Kontakt mit an COVID-19 Erkrankten das Tragen von filtrierenden Halbmasken (FFP2 oder vergleichbar; $[13,23]$ ). Die WHO empfiehlt das Tragen von filtrierenden Masken, wenn Aerosole generiert werden (Übersicht bei [24]). Ebenso ist in der Literatur zu finden: „In conclusion, PPE, when available and properly used, confers protection and lowers infection rates of COVID-19 among health care workers when compared with reported infection rates in the general public" [19].

Unter Gewichtung der Literatur sowie aus den in $\bullet$ Tab. 1 angegebenen Zahlen und Berechnungen kann damit eine Aus- sage über die Eintrittswahrscheinlichkeiten der Gefährdung anhand der modifizierten Risikomatrix nach Nohl [25] vorgenommen werden. Das maximale Schadensausmaß ist dabei bei Eintritt einer COVID-19-Infektion für alle Tätigkeiten einheitlich anzusehen, denn Arbeitsausfall und Dauerschäden sind möglich [26], wohingegen ein tödlicher Verlauf eher unwahrscheinlich ist.

Gemäß der Risikomatrix (• Abb. 2) kann somit unter konsequenter Einhaltung der nach RKI empfohlenen Schutzmaßnahmen [9] und der Maßgaben der TRBA [8] sowie den Informationen des ABAS-Beschlusses von einem mittleren Risiko ausgegangen werden. Dies ist jedoch nur dann gegeben, wenn einerseits adäquate Schutzausrüstung zur Verfügung gestellt wird, andererseits eine bestimmungsgemäße Anwendung sichergestellt ist.

Unsere hier getroffene Risikoabschätzung unterscheidet sich von der Einschätzung des BMAS für besonders Schutzbedürftige [18]. Das BMAS stuft eine Tätigkeit mit bekannten oder vermuteten COVID-19-Erkrankungen und einem mittleren Risiko im Umgang mit infizierten Körperflüssigkeiten als Tätigkeit mit einer hohen Gefährdung und hohem Expositionsrisiko sowie hohem Infektionsrisiko ein. Tätigkeiten im Gesundheitswesen mit bekannter oder vermuteter COVID-19-Erkrankung und einem hohen Risiko im Umgang mit infizierten Körperflüssigkeiten, wie z. B. bei Intubation und Bronchoskopie, werden als Tätigkeit mit einer sehr hohen Gefährdung und einem sehr hohen Infektionsrisiko eingeschätzt [18]. Allerdings werden zu dieser Risikoeinschätzung keine Quellenangaben gemacht. Aufgrund unseres Vergleichs mit der Infektionsrate in der Allgemeinbevölkerung und einer deutlich niedrigeren Todesrate halten wir deshalb die Einschätzung „mittleres Risiko" für gerechtfertigt.

Verschiedenste Tools werden zur Beschreibung von Sicherheitsanalysen eingesetzt, wie „Preliminary Hazard Analysis“, „Fault Tree Analysis“ oder „Failure Mode and Effect Analysis" [27]. Allen diesen Tools ist eigen, dass sie sehr differenziert und dem nicht damit Vertrauten nur schwer verständlich sind [27]. Ge- 


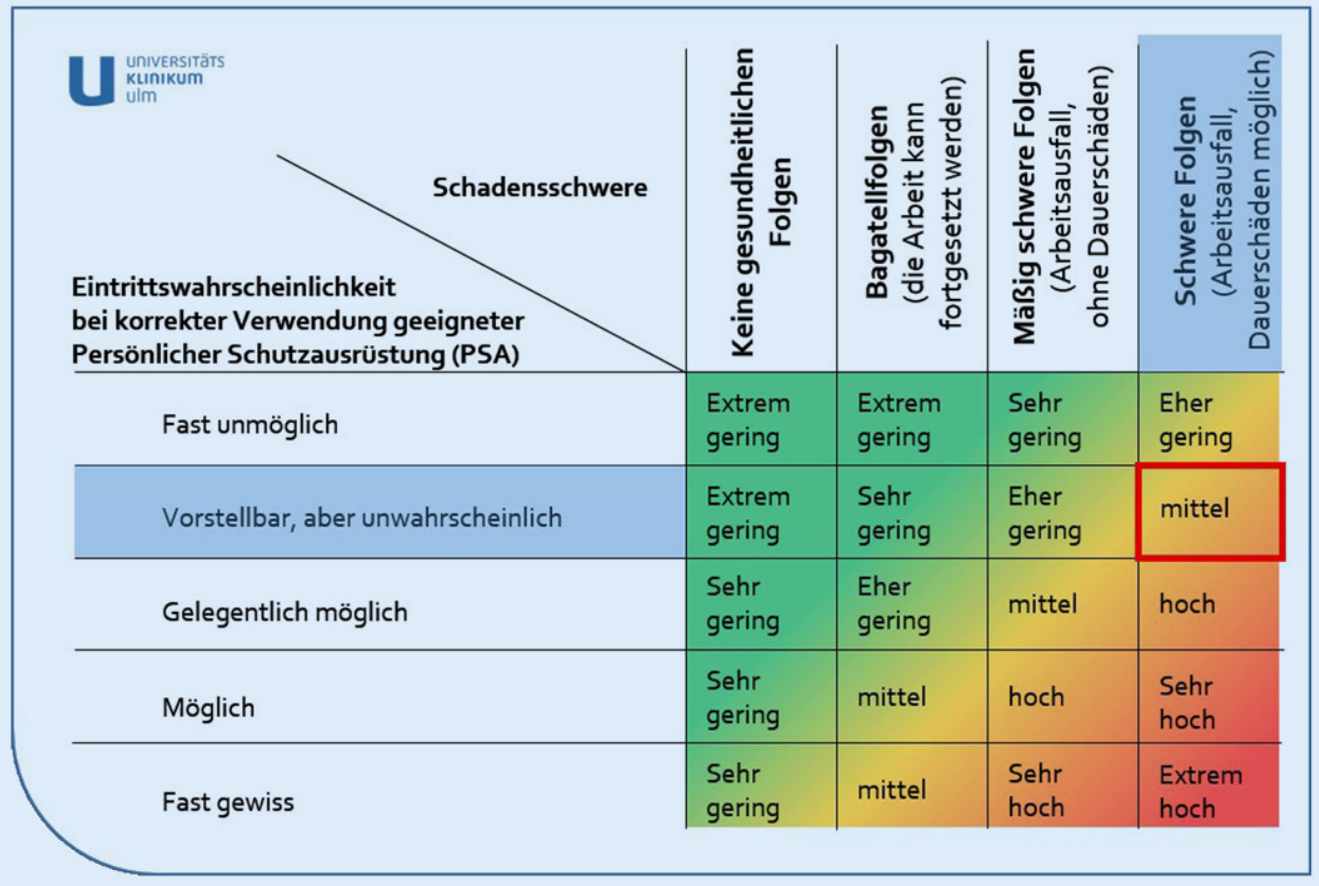

Abb. $2 \varangle$ Risikomatrix adaptiert nach Nohl [24]. Aufgetragen ist die Eintrittswahrscheinlichkeit für medizinisches Personal, an COVID-19 zu erkranken, unter korrekter Verwendung geeigneter persönlicher Schutzausrüstung gegenüber den möglichen Gesundheitsfolgen. Einstufung: rot - hohes Risiko, gelb-mittleres Risiko, grün-geringes Risiko

mäß dem Albert Einstein zugeschriebenen Zitat: „,everything should be made as simple as possible, but not simpler" (zit. nach [28]), empfiehlt sich zur besseren Visualisierung Risikoanalysen als zweidimensionale Matrizen darzustellen, wobei nach ISO 14971 die Eintrittswahrscheinlichkeit gegen die Schwere des Ereignisses aufgetragen wird [28]. Im Arbeitsschutz wird meist die Risikomatrix nach Nohl verwendet [29].

Allerdings lassen sich mit einer zweidimensionalen Matrix nicht alle Aspekte einer Risikoanalyse darstellen. So bleiben Schutzmaßnahmen im Zusammenhang mit COVID-19 wie Unterweisung im An- und Ablegen der PSA, Maßnahmen zur Minimierung der Aerosolbildung bei NIV-Beatmung (nichtinvasive Beatmung), zusätzliche Schutzmaßnahmen bei Intubation, Bronchoskopie und Extubation der beatmeten Patienten [30] unberücksichtigt. Die Risikomatrix lässt auch die erhebliche persönliche Belastung durch das Tragen von Atemmaske und Ganzkörperanzug außer Acht und hierbei vor allem, dass diese zeitabhängig ist. Die Atmung durch FFP2/FFP3Masken ist erheblich erschwert, in den Ganzkörperanzügen kann es durch mangelnde Luftzirkulation zu einem Hitze- stau kommen [31]. In einer chinesischen Studie [32] wurde nachgewiesen, dass die Erkrankung an COVID-19 bei medizinischem Personal mit mangelnder Händedesinfektion und längeren Dienstzeiten assoziiert war. Zudem kann Personal, das mit PSA am Patienten arbeitet für die Tragezeit weder Trinken, Essen noch die Toilette benutzen. Mit zunehmender Tragezeit kann dadurch psychischer Stress entstehen, der zu zunehmender Fehleranfälligkeit und damit Eigen- und Fremdgefährdung führen kann [22]. Im Rahmen der Tätigkeiten, in der Literatur aus anderen Ländern dargestellt in Ausnahmesituation der Triagenotwendigkeit, werden die Beschäftigten mit Situationen konfrontiert, die zu einer psychischen Belastung führen können [33, 34]. Aufgrund der aktuell vorliegenden Lage ist zudem bei Angehörigen, Patienten und potenziellen Besuchern mit Angst- und Stressreaktionen zu rechnen. Verschiedentlich sind dadurch verbale und eventuell auch körperliche Übergriffe auf das Personal möglich.

Die Verwendung geeigneter Atemschutzmasken der Schutzklassen FFP2 bzw. FFP3 ist durch den erhöhten Atemwegswiderstand eine Belastung für das respiratorische System. Es gelten die Vor- gaben für die Benutzung von Atemschutz der Deutschen Gesetzlichen Unfallversicherung (DGUV-Regel 112-190). Infektionshemmende Schutzkleidung ist funktionsbedingt wenig atmungsaktiv (wasserdicht- bzw. abweisend). Der Feuchtigkeitsaustausch mit der Umgebung ist damit eingeschränkt und kann in Abhängigkeit der Umgebungsbedingungen zu einem Wärmestau und einer Dehydrierung führen. Insbesondere bei körperlich anstrengenden Tätigkeiten (bspw. Umlagern von Patienten) führt dies zu einer deutlich erhöhten Belastung des Organismus.

\section{Schutzmaßnahmen (TOP- Prinzip)}

Die im folgenden dargestellten Schutzmaßnahmen gehen über die vom RKI empfohlenen Maßnahmen hinaus und repräsentieren die Umsetzung am Universitätsklinikum Ulm. Für diese zu ergreifenden Schutzmaßnahmen wird auch auf die Handreichung des „Kompetenznetzes Public Health COVID-19“ verwiesen [35]. Zu einem sinnvollen Risikomanagement gehört auch, dass der Risikoeinstufung bzw. -bewertung die Risikobewältigung folgt [36]. Gemäß $\$ 4$ 


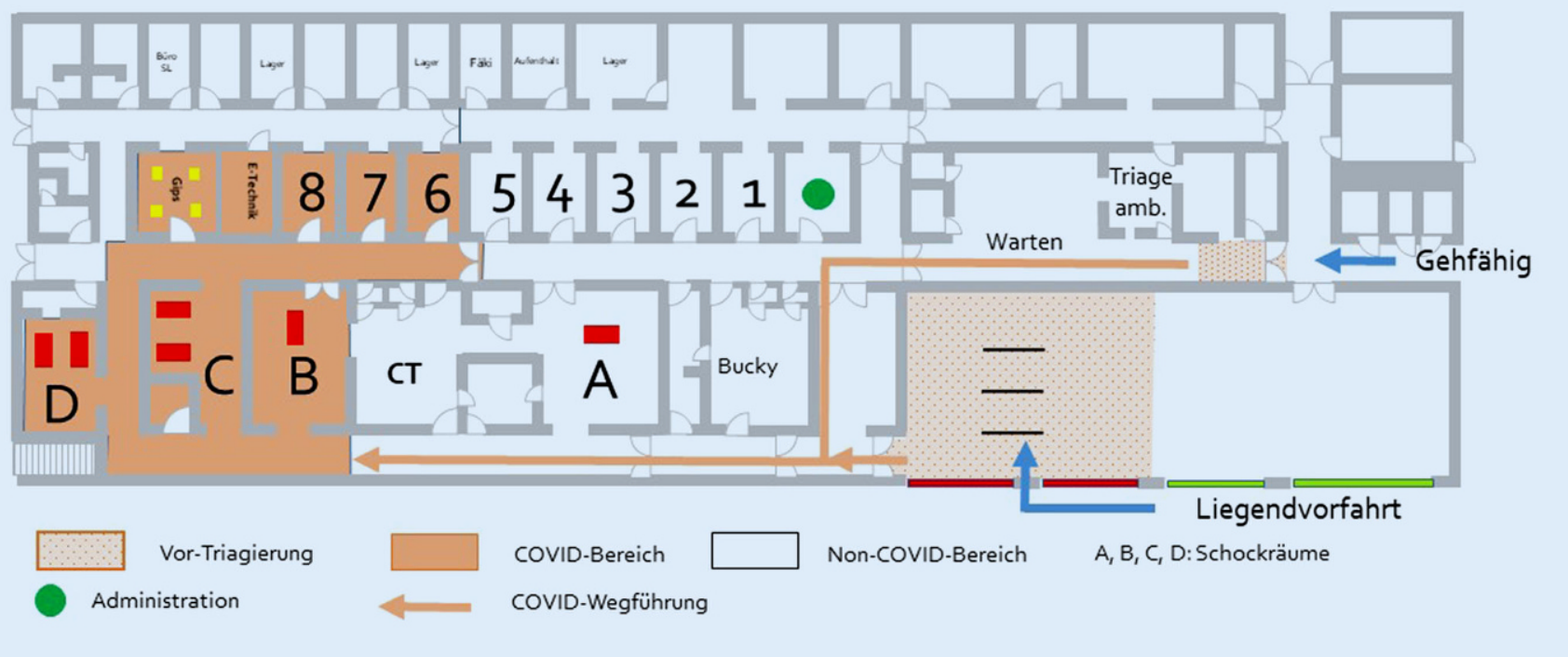

Abb. $3 \triangle$ Aufteilung einer zentralen interdisziplinären Notaufnahme in einen COVID- und einen Non-COVID-Bereich. Die Großbuchstaben markieren die 4 Schockräume, wobei in B, C und D COVID-19-Patienten erstversorgt werden. Die Zahlen 1 bis 8 markieren die Behandlungsräume, 6 bis 8 für COVID-19-Patienten. In der obersten Reihe befinden sich im linken Bereich die administrativen Räume und rechts die Kurzbehandlungszimmer (Decision Unit)

Arbeitsschutzgesetz müssen Gefahren immer direkt an der Quelle beseitigt oder entschärft werden, und zwar nach dem „TOP-Prinzip“ (technische Maßnahmen, organisatorische Maßnahmen, personenbezogene Maßnahmen).

\section{Technische Maßnahmen}

An oberster Stelle der Maßnahmenhierarchie wird grundsätzlich die Vermeidung von Emissionen - hier die Virenausbreitung - priorisiert. Die größte Wirksamkeit entfaltet sich bei Interventionen direkt am Entstehungsort. Hierzu bieten sich folgende technische Möglichkeiten:

Sofern technisch möglich, sollten im Raum Unterdruckverhältnisse gegeben sein, um eine Verteilung des tröpfchenoder aerosolgetragenen COVID-19Erregers zu unterbinden. Bei provisorischen Umnutzungen ist auf unerwünschte Luftströmungen in nichtkontaminierte Bereiche (Weißbereiche) zu achten. Aus krankenhaushygienischer Sicht ist eine vorgeschaltete Schleusenfunktion sinnvoll. Ergänzend können UmluftGeräte mit einer endständigen HEPAFilterstufe (High Efficency Particulate Air Filter) eingesetzt werden [22], um die Viruskonzentration in der Raumluft zusätzlich zu reduzieren. Dies ersetzt in keinem Fall den Einsatz von partikel- filternden Halbmasken im Rahmen der persönlichen Schutzausausrüstung.

\section{Organisatorische Maßnahmen}

Um Beschäftigte vor einer Infektion bei der Arbeit mit SARS-CoV-2 soweit als möglich zu schützen, sind vielerorts Neu- und Umgestaltungen von Arbeitsplätzen und Abläufen notwendig [22]. Eine infektionspräventive Triagierung ist bereits am Klinikeingang und noch vor der Aufnahme des Patienten notwendig. Die konsequente Trennung im weiteren Verlauf - Anamnese, Behandlung sowie stationäres Umfeld ist unerlässlich. Ein Wechsel von Mitarbeiter ${ }^{\star}$ innen zwischen COVID-Bereichen und Non-COVID-Bereichen ist zu vermeiden. Patientenkontakte dürfen nur unter Berücksichtigung des Minimierungsgebotes sowie bereitgestellter persönlicher Schutzkleidung erfolgen. Zur Vermeidung einer Kontaminationsverschleppung muss die kontaminierte Schutzkleidung fachgerecht abgelegt und in dafür vorgesehene Behältnisse entsorgt werden. Abschließend erfolgt eine hygienische Händedesinfektion. Zusätzlich sind die Vorgaben des Hautschutzplanes seitens des Betriebsärztlichen Dienstes $\mathrm{zu}$ beachten. Die strikte Trennung von Arbeits- und Pausenbereichen ist einzuhalten. Die Einnahme von Lebensmitteln außerhalb der Pausenbereiche ist verboten. Mindestabstände ( $>1,5 \mathrm{~m}$ ) sowie Vermeidung von Gruppenbildung an den Arbeitsplätzen sowie in Pausen- und Umkleideräumen können die Gefahr der Infektionsweitergabe minimieren [22]. Beispielhaft ist in - Abb. $\mathbf{3}$ die organisatorische Trennung in einen COVID- und Non-COVID-Bereich der Zentralen Interdisziplinären Notaufnahme der Universitätsklinik Ulm dargestellt. Nicht immer lassen sich kreuzende Wegführungen zwischen COVID- und Non-COVID-Patienten vermeiden. Hier kann das Infektionsrisiko durch Wegemarkierungen auf dem Fußboden sowie vermehrte Desinfektion und Lüftung reduziert werden [22].

Kontaktstellen (Türklinken, Griffflächen von Geräten etc.) erfordern bei der Flächendesinfektion besonderes Augenmerk. Wischdesinfektion ist der Sprühdesinfektion generell vorzuziehen. Ist dies nicht möglich, muss auf eine konsequente Lüftung des Bereichs geachtet werden. Der Luftwechsel ist durch natürliche oder technische Lüftung so zu erhöhen, dass Viren mit dem Luftstrom fortgetragen werden können.

Die Unterweisung und Qualifizierung von Mitarbeiter*innen für besondere oder neu übertragene Tätigkeiten 
(z.B. Bedienung Beatmungsgeräte oder Verwendung ungewohnter PSA) muss über die Vorgesetzten organisiert und schriftlich dokumentiert werden.

Zugänge zu Kliniken werden dahin gehend gesichert, dass der Publikumsverkehr für Besucher eingestellt wird. Im Rahmen sinkender Infektionszahlen kann eine schrittweise Lockerung dieser Besuchsregelung vorgenommen werden. Kliniken können auch für spezielle Bereiche wie Kinderklinik oder Geburtsabteilungen spezielle Sonderregelungen erlassen. Der Zugang für Patienten ist über wenige und durch den Sicherheitsdienst überwachte Eingänge möglich. Gleiches gilt für Nebeneingänge, die nur für das Klinikpersonal freigegeben sind. Beim Betreten des Gebäudes wird zudem eine Temperaturmessung durchgeführt, wenngleich diese Maßnahme umstritten ist; die Mitarbeiter ${ }^{*}$ innen werden gebeten eine Händedesinfektion vorzunehmen.

Mitarbeitende, Patienten und eventuelle Besuchende sind beim Betreten der Kliniken verpflichtet einen Mund-Nasen-Schutz (MNS) anzulegen. Dies gilt auch, wenn kein direkter Patientenkontakt zu erwarten ist.

Tätigkeiten mit infizierten oder infektionsverdächtigen Patienten sowie in kontaminierten Bereichen sind nur unter Verwendung von PSA zulässig. Zur Erreichung des Schutzniveaus darf diese nur gemäß Herstellergebrauchshinweisen eingesetzt werden. Sollte geeignete PSA nicht mehr zur Verfügung stehen, sind Ersatzmaßnahmen jeweils mit dem Vorgesetzten abzustimmen.

Es wird empfohlen, COVID-19-Patientenzimmer/Untersuchungszimmer nur in vollständiger PSA zu betreten. Sie besteht aus [9]: flüssigkeitsdichtem Schutzkittel, Handschuhen, Maske - Schutzstufe FFP2 oder mehr - bei Tätigkeiten mit starker Aerosolbildung, Kopfhaube und geeignete Schutzbrille. Bei der Betreuung mit COVID-19-Patienten mit NIV/High-Flow wird eine PSA bestehend aus Ganzkörperoverall mit Kopfhaube, Handschuhen, Maske - Schutzstufe FFP2 oder mehr - und Schutzbrille/Gesichtsvisier empfohlen (siehe auch [35]).
Für patientennahe Tätigkeiten ist mindestens eine Schutzbrille, bei invasiven Eingriffen und Untersuchungen (z.B. Bronchoskopie, Intubation, Tracheotomie) ist zusätzlich ein Gesichtsvisier $\mathrm{zu}$ verwenden. Innerhalb der Kliniken besteht für alle Mitarbeiter*innen die Pflicht, Mund-NasenSchutz zu tragen. Bei direkter Versorgung von Patienten mit bestätigter oder wahrscheinlicher COVID-19-Erkrankung sollten bevorzugt FFP2-Masken getragen werden (Schutz vor Aerosolen und Tröpfchen). Bei allen Tätigkeiten, die mit Aerosolproduktion einhergehen (z.B. Intubation oder Bronchoskopie), sollen Atemschutzmasken (FFP2 oder darüber hinausgehender Atemschutz) getragen werden.

Um Kontaktinfektionen in der $\mathrm{Pa}$ tientenversorgung (Querinfektionen) $\mathrm{zu}$ vermeiden, tragen die Mitarbeiter*innen, die Kontakt zu infizierten Patienten haben, Bereichskleidung und geeignete Schutzkittel, Hauben, Schutzbrille/Visier und Schutzhandschuhe. Bei allen Tätigkeiten, die mit Aerosolproduktion einhergehen (z.B. bei NIV/ High-Flow, Intubation, Bronchoskopie), sollen flüssigkeitsdichte Overalls mit Eignung gegen biologische Agenzien getragen werden. Zusätzlich sind Schutzhandschuhe zu tragen.

\section{Würdigung der umgesetzten Maßnahmen}

Krankenhäuser zählen zu den kritischen Infrastrukturen, die per definitionem als Organisationen und Einrichtungen mit wichtiger Bedeutung für das staatliche Gemeinwesen anzusehen sind, bei deren Beeinträchtigung nachhaltig wirkende Versorgungsengpässe, erhebliche Störungen der öffentlichen Sicherheit oder andere dramatische Folgen eintreten würden [37]. „Der Schutz, der im Krankenhaus arbeitenden bzw. versorgten Personen, muss deshalb für alle relevanten internen und externen Schadenslagen höchste Priorität haben“" [37]. Dieses anzustrebende Ziel allein impliziert schon die Notwendigkeit eines effektiven Risikomanagements für COVID-19.
Unsere empfohlenen Schutzmaßnahmen gehen daher über die Empfehlungen des RKI hinaus (• Tab. 2). Sie lassen sich teils aus neueren Publikationen ableiten, aber auch aus dem ethischen Gebot des maximalen Schutzes für Personal und $\mathrm{Pa}$ tienten. Absatz 3(6) der SARS-CoV-2Arbeitsschutzregel [22] besagt: „Es ist zu prüfen, ob und inwieweit für besonders schutzbedürftige Beschäftigte zusätzlich zu kollektiven Maßnahmen individuelle Maßnahmen zum Schutz vor einer Ansteckung durch Beschäftigte oder Kunden zu treffen sind." Das Bundesministerium für Arbeit und Soziales hat einen sehr detaillierten Katalog an Erkrankungen und Beeinträchtigungen veröffentlicht, die das Risiko, an SARS-CoV-2 zu erkranken, erhöhen [18]. Da Erkrankungen und Beeinträchtigungen der ärztlichen Schweigeplicht unterliegen und der Arbeitgeber nicht berechtigt ist darüber Auskunft zu erlangen, sie sich zeitlich ändern können und die individuelle Umsetzung nur mit erheblichem logistischen Aufwand möglich wäre, hat sich das Universitätsklinikum Ulm für einen erweiterten Schutz entschlossen.

Eine Gruppe von 239 Wissenschaftler*innen hat kürzlich einen offenen Brief unterzeichnet, in dem die Weltgesundheitsorganisation und andere internationale Einrichtungen des öffentlichen Gesundheitswesens aufgefordert werden, zusätzliche Vorsichtsmaßnahmen zum Schutz vor potenzieller Übertragung durch die Luft zu empfehlen [39]. Zudem konnten in einer als Preprint veröffentlichten Mitteilung Lednicky et al. zeigen, dass infizierte Patienten in ihren Zimmern in der Klinik virenhaltige Aerosole weiter als bisher vermutet verbreiten: „Our findings reveal that viable SARS-CoV-2 can be present in aerosols generated by a COVID-19 patient in a hospital room in the absence of an aerosol-generating procedure, and can thus serve as a source for transmission of the virus in this setting ... For aerosolbased transmission, measures such as physical distancing by 6 feet would not be helpful in an indoor setting, provide a false-sense of security and lead to exposures and outbreaks" [38].

Morawska et al. warfen in einem Übersichtsartikel die Frage auf: „How can air- 
Tab. 2 Vergleichende Darstellung der vom Robert Koch-Institut (RKI) empfohlenen Schutzmaßnahmen und den nach Meinung der Autoren zu ergreifenden Maßnahmen beim Umgang mit COVID-19-Patienten für Personal mit direktem Kontakt zu COVID-19-Patienten

\begin{tabular}{|c|c|c|c|}
\hline \multicolumn{2}{|c|}{ Vergleichende Darstellung der Schutzmaßnahmen nach TOP } & \multirow{2}{*}{$\begin{array}{l}\text { RKI-Emp- } \\
\text { fehlung [9] }\end{array}$} & \multirow{2}{*}{$\begin{array}{l}\text { Empfohlene } \\
\text { Maßnahmen }\end{array}$} \\
\hline $\begin{array}{l}\text { Technische } \\
\text { Maßnahmen }\end{array}$ & $\begin{array}{l}\text { Anpassung raumlufttechnischer Anlagen zur } \\
\text { Vermeidung einer Verbreitung des Erregers }\end{array}$ & & \\
\hline & $\begin{array}{l}\text { Anpassung der Raumdruckverhältnisse (Unter- } \\
\text { druck) }\end{array}$ & - & $\mathrm{x}$ \\
\hline & $\begin{array}{l}\text { Partielle Verwendung von HEPA-Umluftfiltern } \\
\text { im Raum }\end{array}$ & - & $\mathrm{x}$ \\
\hline \multirow{6}{*}{$\begin{array}{l}\text { Organisa- } \\
\text { torische Maß- } \\
\text { nahmen }\end{array}$} & $\begin{array}{l}\text { Trennung von Non-COVID-19- und COVID-19- } \\
\text { Patienten }\end{array}$ & $\mathrm{x}$ & $x$ \\
\hline & Nutzung von Isolierzimmern mit Schleuse & $x$ & $x$ \\
\hline & Einsatz unterwiesenen Personals & $x$ & $x$ \\
\hline & $\begin{array}{l}\text { Ergänzende Unterweisungen zur persönlichen } \\
\text { Schutzausrüstung (PSA) durch Vorgesetzte }\end{array}$ & - & $\mathrm{x}$ \\
\hline & Entsorgung als infektiöser Müll & - & $\mathrm{x}$ \\
\hline & Wischdesinfektion patientennaher Flächen & x (täglich) & $\begin{array}{l}x \text { (mehrmals } \\
\text { täglich) }\end{array}$ \\
\hline \multirow{10}{*}{$\begin{array}{l}\text { Persönliche } \\
\text { Schutzausrüs- } \\
\text { tung }\end{array}$} & $\begin{array}{l}\text { Mund-Nasen-Schutz (MNS) für Tätigkeiten ohne } \\
\text { erhöhtes Infektionsrisiko im gesamten Klinikum }\end{array}$ & $\mathrm{x}$ & $\mathrm{x}$ \\
\hline & $\begin{array}{l}\text { FFP2-Maske für Tätigkeiten mit COVID-19-Pati- } \\
\text { enten }\end{array}$ & $\mathrm{x}$ & $x$ \\
\hline & $\begin{array}{l}\text { Mindestens FFP2-Maske für Tätigkeiten mit } \\
\text { Aerosolproduktion }\end{array}$ & $\mathrm{x}$ & $\mathrm{x}$ \\
\hline & Filtergeräte mit Gebläseunterstützung & - & $\mathrm{x}$ \\
\hline & Schutzkittel MRSA & $\mathrm{x}$ & $\mathrm{x}$ \\
\hline & $\begin{array}{l}\text { Schutzoverall für Tätigkeiten mit Aerosolproduk- } \\
\text { tion }\end{array}$ & - & $\mathrm{x}$ \\
\hline & Schutzbrille & $\mathrm{x}$ & $\mathrm{x}$ \\
\hline & $\begin{array}{l}\text { Zusätzlich Schutzvisier für Tätigkeiten mit Aero- } \\
\text { solproduktion }\end{array}$ & - & $x$ \\
\hline & Einweghandschuhe & $x$ & $\mathrm{x}$ \\
\hline & Double-Gloving in Risikobereichen & - & $x$ \\
\hline
\end{tabular}

born transmission of COVID-19 indoors be minimised?" Die Autoren schlagen vor, die vorhandenen Lüftungssysteme mit portablen Reinigungssystemen zu ergänzen, die mit mechanischen Filtern ausgestattet sind [40]. Sie resümieren: „While much of the focus has been on case finding, isolation and quarantine, social distancing and hand hygiene, we emphasise that a parallel reduction in airborne transmission using such engineering controls in hospitals and other public buildings will further protect healthcare workers, patients and the general public." Miller et al. wiesen zudem auf die Möglichkeit hin, auf Infektionsstationen Systeme zur Unterdruckerzeugung einzubauen [41].
Gegebenheiten auch für andere Kliniken hilfreich sein.

Die SARS-CoV-2-Pandemie ist in Deutschland und weltweit ein sehr dynamisches Geschehen [17] mit bisher noch ungenügenden Erkenntnissen, fortlaufend werden neue Forschungsergebnisse publiziert. Damit handelt es sich bei der hier dargestellten Einschätzung und den daraus abgeleiteten Schussfolgerungen um eine Status-quo-Darstellung, die durch neuere Erkenntnisse eventuell revidiert werden muss.

Eine weitere Einschränkung ergibt sich aus der Ungenauigkeit der Schätzung des in der Bundesrepublik Deutschland tätigen medizinischen Personals. Zum einen stammen die letzten Angaben des Statistischen Bundesamts aus 2018 und diese auch nur als vorläufige Zahlen [15]. Zum anderen sind die Angaben des RKI bezüglich Infektionen und Todesfälle auf die im Gesamtbereich des medizinischen Sektors Tätigen bezogen (Krankenhäuser, ärztliche Praxen, Dialyseeinrichtungen und Rettungsdienste); eine Differenzierung der speziell in Kliniken Tätigen ist nicht möglich. Zudem gibt das RKI an: „Da Angaben zu Betreuung, Unterbringung und Tätigkeit bei $25 \%$ der Fälle fehlen, sind die Anteile der Fälle mit einer Betreuung, Unterbringung oder Tätigkeit in den einzelnen Einrichtungen als Mindestangaben zu verstehen. Für die übermittelten COVID19-Fälle aus allen genannten Einrichtungen ist jedoch unbekannt, wie hoch der Anteil derer ist, die sich auch in dieser Einrichtung angesteckt haben“ [11].

\section{Schussfolgerungen}

dass die hier dargestellten Einschätzungen und Empfehlungen der Autoren sich zwar aus dem Regelwerk und den biologischen Übertragungsmechanismen $\mathrm{zu}$ COVID-19 ableiten lassen, aber auch auf eigenen Erfahrungen beruhen. Viele Entscheidungen über die infektionspräventiven Arbeitsschutzmaßnahmen nach dem TOP-Prinzip müssen aus der Praxis entwickelt werden. Dargestellt ist somit ein evidenzinformierender Leitfaden der Universitätsklinik Ulm sowie dessen Umsetzung. Dieser Leitfaden kann unter Berücksichtigung lokaler
- Das Infektionsrisiko für ungeschütztes medizinisches Personal, an COVID-19 zu erkranken, ist laut Bundesministerium für Arbeit und Soziales je nach Tätigkeitsfeld mit hoch bis sehr hoch einzustufen. Das tatsächliche Risiko, mit der Infektionsmöglichkeit außer- und innerklinisch, entspricht trotz hoher Patientenkontakte und Kontaktzeiten dagegen dem Risiko in der Gesamtbevölkerung, dieses wird Ende August 2020 vom RKI als „hoch“ eingeschätzt. 
- Verglichen mit der Allgemeinbevölkerung ist in Deutschland die Infektrate des medizinischen Personals deutlich niedriger als in anderen Ländern [42]. Die Mortalitätsrate des medizinischen Personals beträgt in Deutschland nur einen Bruchteil der Mortalitätsrate der Gesamtbevölkerung. Dies ist spekulativ nicht nur dem niedrigeren Durchschnittsalter des medizinischen Personals und der damit verbundenen milderen Ausprägung der COVID-19-Erkrankung geschuldet, sondern vor allem der Aussetzung einer geringeren Viruslast durch die sachgerechte Verwendung einer PSA.

- Medizinisches Personal, das im direkten Kontakt zu COVID-19-Patienten steht, muss deshalb konsequent durch eine gefährdungsadaptierte PSA geschützt werden. Mit entsprechender PSA und deren korrekter Anwendung ist das Infektionsrisiko für medizinisches Personal als „mittel“ einzustufen.

\section{Korrespondenzadresse}

\section{Prof. Dr. Ernst G. Pfenninger}

Stabsstelle Katastrophenschutz, Universitätsklinikum Ulm

Albert-Einstein-Allee 29, 89071 Ulm,

Deutschland

ernst.pfenninger@uniklinik-ulm.de

\section{Einhaltung ethischer Richtlinien}

Interessenkonflikt. E.G. Pfenninger, P. Christ, M. Neumüller und A. Dinse-Lambracht geben an, dass kein Interessenkonflikt besteht.

Für diesen Beitrag wurden von den Autoren keine Studien an Menschen oder Tieren durchgeführt. Für die aufgeführten Studien gelten die jeweils dort angegebenen ethischen Richtlinien.

\section{Literatur}

1. World Health Organization World Health Organization. Coronavirus disease (COVID-19) Situation Report. https://covid19.who.int/. Zugegriffen: 27. Aug. 2020

2. The New York Times Virus knocks thousands of health workers out of action in Europe (24.03.2020). https://www.nytimes.com/2020/ 03/24/world/europe/coronavirus-europe-covid19.html. Zugegriffen: 18. Aug. 2020
3. Zhonghua Liu Xing Bing Xue Za Zhi . (2020) The epidemiological characteristics of an outbreak of 2019 novel coronavirus diseases (COVID-19) in China. [Abstract in English]. http://html.rhhz.net/ zhlxbx/004.htm https://doi.org/10.3760/cma.j. issn.0254-6450.2020.02.003.Zugegriffen: 18. Aug. 2020

4. CDC COVID-19 Response Team (2020) Characteristics of health care personnel with COVID19-United States, February 12-April 9, 2020. MMWR Morb Mortal Wkly Rep 69:477-481

5. Mitchell R, Ogunreai T, Astratianakis S, Bryce E, Gervais R, Gravel D, Johnson L, Leduc S, Roth V, Taylor G, Vearcombe M, Weir C (2012) Impact of the 2009 influenza A (H1N1) pandemic on Canadian health care workers: a survey on vaccination, illness, absenteeism, and personal protective equipment. Am J Infect Control 40:611-616

6. Buda S, an der Heiden M, Altmann D, Diercke M, Hamouda O, Rexroth U (2020) Infektionsumfeld von erfassten COVID-19-Ausbrüchen in Deutschland. Epid Bull 38:3-12. https://doi.org/10.25646/7093

7. Robert Koch Institut SARS-CoV-2 Steckbrief zur Coronavirus-Krankheit-2019 (COVID-19). Stand: 21.8.2020. https://www.rki.de/DE/Content/InfAZ/ N/Neuartiges_Coronavirus/Steckbrief.html\# doc13776792bodyText4. Zugegriffen: 27. Aug. 2020

8. Beschluss des $A B A S$ zur vorläufigen Einstufung des Virus SARS-CoV-2 in Risikogruppe 3 und Empfehlungen zu nicht gezielten Tätigkeiten (Labordiagnostik) und gezielten Tätigkeiten mit SARS-CoV-2. Stand: 4. Juni 2020 https://www. baua.de/DE/Aufgaben/Geschaeftsfuehrungvon-Ausschuessen/ABAS/pdf/SARS-CoV-2.pdf? blob=publicationFile\&v=3. Zugegriffen: 27. August 2020

9. Robert Koch Institut Empfehlungen des RKI zu Hygienemaßnahmen im Rahmen der Behandlung und Pflege von Patienten mit einer Infektion durch SARS-CoV-2 Stand 5.6.2020. https://www.rki.de/ DE/Content/InfAZ/N/Neuartiges_Coronavirus/ Hygiene.html?nn=13490888. Zugegriffen: 18. Aug. 2020

10. The national academies of Sciences, Engineering, Medicine (2020) Rapid expert consultation on the possibility of bioaerosol spread of SARS-coV-2 for the COVID-19 pandemic (April 1, 2020). https://www.nap.edu/read/25769/ chapter/1.Zugegriffen: 18. Aug. 2020

11. Täglicher Lagebericht des RKI zur CoronavirusKrankheit-2019 (COVID-19) vom 16.04.2020. https://www.rki.de/DE/Content/InfAZ/N/ Neuartiges_Coronavirus/Situationsberichte/ 2020-04-16-de.pdf?_blob=publicationFile. Zugegriffen:27. Aug. 2020

12. BGW Biostoffe. https://www.bgw-online. de/DE/Arbeitssicherheit-Gesundheitsschutz/ Gefaehrdungsbeurteilung/Biostoffe/Biostoffe. html.Zugegriffen: 27. Aug. 2020

13. Robert Koch Institut Optionen zum Managemen von Kontaktpersonen unter Personal der kritischen Infrastruktur bei Personalmangel. https:// www.rki.de/DE/Content/InfAZ/N/Neuartiges_ Coronavirus/Personal_Kritls.html.Zugegriffen: 18. Aug. 2020

14. Deutsche Gesellschaft für Krankenhaushygiene e. V. Luftqualität im OP-Saal: Wundinfektionen, RLT-Anlagen und Disziplin. https://www. krankenhaushygiene.de/ccUpload/upload/files/ 2018_11_13_DGKH-Stellungnahme_Luftqualit \%C3\%A4t-im-OP-Saal.pdf. Zugegriffen: 18. Aug. 2020
15. Statistisches Bundesamt (2020) Gesundheitspersonal. https://www.destatis.de/DE/ Themen/Gesellschaft-Umwelt/Gesundheit/ Gesundheitspersonal/_inhalt.html. Zugegriffen: 18. Aug. 2020

16. Täglicher Lagebericht des RKI zur CoronavirusKrankheit-2019 (COVID-19) vom 18.06.2020. https://www.rki.de/DE/Content/InfAZ/N/ Neuartiges_Coronavirus/Situationsberichte/ 2020-06-18-de.pdf?_blob=publicationFile. Zugegriffen:22. Aug. 2020

17. Täglicher Lagebericht des RKI zur CoronavirusKrankheit-2019 (COVID-19) vom 21.08.2020. https://www.rki.de/DE/Content/InfAZ/N/ Neuartiges_Coronavirus/Situationsberichte/ 2020-08-21-de.pdf?_blob=publicationFile. Zugegriffen:27.Aug. 2020

18. Bundesministerium für Arbeit und Soziales Umgang mit aufgrund der SARS-CoV-2-Epidemie besonders schutzbedürftigen Beschäftigten. Arbeitsmedizinische Empfehlungen. https:// www.bundesregierung.de/breg-de/service/ publikationen/umgang-mit-aufgrund-der-sarscov-2-epidemie-besonders-schutzbeduerftigenbeschaeftigten-1770362. Zugegriffen: 22 . Aug. 2020

19. Jeremias A, Nguyen J, Levine J et al (2020) Prevalence of SARS-CoV-2 infection among health care workers in a tertiary community hospital. JAMA Intern Med. https://doi.org/10. 1001/jamainternmed.2020.4214

20. Wang X, Ferro EG, Zhou G, Hashimoto D, Bhatt DL (2020) Association between universal masking in a health care system and SARS-coV-2 positivity among health care workers. JAMA 324:703-704. https://doi.org/10.1001/jama.2020.12897

21. Brooks JT, Butler JC, Redfield R (2020) Universal masking to prevent SARS-coV-2 transmission - the time is now. JAMA 324:635-637. https:// doi.org/10.1001/jama.2020.13107

22. Bhaskar ME, Arun S (2020) SARS-coV-2 infection among community health workers in India before and after use of face shields.JAMA. https://doi.org/ 10.1001/jama.2020.15586

23. SARS-CoV-2-Arbeitsschutzregel. (Fassung 20.08.2020). Arbeitsausschüsse beim BMAS https://www.baua.de/DE/Angebote/Rechtstexteund-Technische-Regeln/Regelwerk/AR-CoV-2/ pdf/AR-CoV-2.pdf?_blob=publicationFile\& $\mathrm{v}=10$. Zugegriffen: 20. Aug. 2020

24. Park SH (2020) Personal protective equipment for Healthcare workers during the COVID-19 pandemic. Infect Chemother 52:165-182. https:// doi.org/10.3947/ic.2020.52.2.165

25. Nohl J, Thiemecke H (1988) Systematik zur Durchführung von Gefährdungsanalysen, Teil I und II. (Schriftenreihe der Bundesanstalt für ArbeitsschutzFb536undFb542. Wirtschaftsverlag NW, Bremerhaven

26. Carfi A, Bernabei R, Landi F et al (2020) Persistent symptoms in patients after acute COVID-19. JAMA 324:603-605. https://doi.org/10.1001/jama.2020. 12603

27. Watson C Risk assessment using the three dimensions of probability (likelihood), severity, and level of control. NASA technical reports server. https:// ntrs.nasa.gov/search.jsp?R=20100024129. Zugegriffen: 18.Aug. 2020

28. Dickson J (2011) On measures for illustrating credit risk assessments: the case of heat maps, risk matrices and cubes. In: Proceedings of the IFC Conference on "Initiatives to address data gaps revealed by the financial crisis" 25.-26. Aug 2010. 
Bd. 34. Bank for International Settlements, Basel, S365-372

29. Müller N, Arenz T (2011) Sichere Lagerung gefährlicher Stoffe: von der Theorie zur Praxis, 6. Aufl. Hüthing Jehle Rehm, Heidelberg, München, Landsberg, Frechen, Hamburg, S 117

30. Kluge S, Janssens U, Welte T et al (2020) Empfehlungen zur intensivmedizinischen Therapie von Patienten mit COVID-19. Med Klin Intensivmed Notfmed 115:175-177. https://doi.org/10.1007/ s00063-020-00674-3

31. DIVI Schutz und Erhalt der psychischen Gesundheit von Mitarbeitern in Notaufnahmen und auf Intensivstationen während der COVID-19-Pandemie. https://www.divi.de/ empfehlungen/publikationen/covid-19/1547schutz-und-erhalt-der-psychischen-gesundheitvon-mitarbeitern-in-notaufnahmen-und-aufintensivstationen-waehrend-der-covid-19 pandemie.Zugegriffen: 18. Aug. 2020

32. Ran L, Chen X, Wang Y, Wu W, Zhang L, Tan X (2020) Risk factors of healthcare workers with corona virus disease 2019: a retrospective cohort study in a designated hospital of Wuhan in China. Clin Infect Dis. https://doi.org/10.1093/cid/ciaa287

33. Zhang W, Wang K, Yin L, Zhao W, Xue Q et al (2020) Mental health and psychosocial problems of medical health workers during the COVID-19 epidemic in China. Psychother Psychosom. https:// doi.org/10.1159/000507639

34. Zhao N, Huang Y (2020) Chinese mental health burden during COVID-19 outbreak: a web-based cross-sectional survey. Asian J Psychol. https://doi. org/10.1016/j.ajp.2020.102052

35. Kämpf D, Bolm-Audorff U, Petereit-Haack G, Stranzinger J (2020) Kompetenznetz Public Health COVID-19. COVID-19 und Arbeitsschutz im Gesundheitswesen. Technische, organisatorische und persönliche Schutzmaßnahmen zur Verhütung einer Infektion mit SARS-CoV-2 bei Beschäftigten im Gesundheitswesen. https:// www.public-health-covid19.de/images/2020/ Ergebnisse/COVID-19_und_Arbeitsschutz_im Gesundheitswesen_01.pdf.Zugegriffen: 27. Aug. 2020

36. Exner K, Hölzl M (2017) Risikobewertung: Methoden und Herausforderungen bei der Bewertung von Risiken im unternehmensweiten Risikomanagement. https://www.3grc.de/ risikomanagement/risikobewertung-methodenund-herausforderungen-bei-der-bewertungvon-risiken-im-unternehmensweiten-risiko management/.Zugegriffen: 20. Aug. 2020

37. Bundesamt für Bevölkerungsschutz und Katastrophenhilfe (2008) Schutz kritischer Infrastruktur: Risikomanagement im Krankenhaus. In: Praxis im Bevölkerungsschutz - Band 2. https:// www.bbk.bund.de/SharedDocs/Downloads/BBK/ DE/Publikationen/Praxis_Bevoelkerungsschutz/ Band_2_Risikoman_Krankh_Leitfaden_Auszug_ CD-ROM.htm. Zugegriffen: 18. Aug. 2020

38. Lednicky JA, Lauzardo M, Fan ZH, Jutla AS, Tilly TB, Gangwar M et al (2020) Viable SARS-CoV-2 in the air of a hospital room with COVID-19 patients. Preprint 03.08.2020.BMJ.https://doi.org/10.1101/ 2020.08.03.20167395

39. Morawska L, Milton DK (2020) It is time to address airborne transmission of COVID-19. Clin Infect Dis. https://doi.org/10.1093/cid/ciaa939

40. Morawska L, Tang JW, Bahnfleth W, Bluyssend PMet al (2020) How can airborne transmission of COVID19 indoors be minimised? Environ Int 142:105832. https://doi.org/10.1016/j.envint.2020.105832
41. Miller SL, Clements N, Elliott SA, Subhash SS, Eagan A, Radonovich LJ (2017) Implementing a negative-pressure isolation ward for a surge in airborne infectious patients. Am J Infect Control 45:652-659. https://doi.org/10.1016/j. envint.2020.105832

42. Ngyen LH, Drew DA, Graham MS, Joshi AD, Guo CG, Ma W et al (2020) Risk of COVID-19 among front-line health-care workers and the general community: a prospective cohort study. Lancet 5:E475-E483. https://doi.org/10.1016/S24682667(20)30164-X 